\title{
Fixed points and approximately octic mappings in non-Archimedean 2-normed spaces
}

\author{
Choonkil Park' ${ }^{1}$ Madjid Eshaghi Gordji ${ }^{2 *}$, Mohammad Bagher Ghaemi ${ }^{3}$ and Hamid Majani ${ }^{3}$
}

\section{"Correspondence:}

madjid.eshaghi@gmail.com

2Department of Mathematics,

Semnan University, P.O. Box

35195-363, Semnan, Iran

Full list of author information is

available at the end of the article

\begin{abstract}
Using the fixed point method, we investigate the Hyers-Ulam stability of the system of additive-cubic-quartic functional equations with constant coefficients in non-Archimedean 2-normed spaces. Also, we give an example to show that some results in the stability of functional equations in (Archimedean) normed spaces are not valid in non-Archimedean normed spaces.
\end{abstract}

MSC: 39B82; 46S10; 39B52; 47S10; 47H10

Keywords: octic functional equation; Hyers-Ulam stability; non-Archimedean 2-normed space; fixed point method

\section{Introduction}

Gähler [1,2] has introduced the concept of linear 2-normed spaces. Then Gähler and White [3-5] introduced the concept of 2-Banach spaces. In 1999 to 2003, Lewandowska published a series of papers on 2-normed sets and generalized 2-normed spaces [6, 7]. Recently, Park [8] investigated approximate additive mappings, approximate Jensen mappings and approximate quadratic mappings in 2-Banach spaces. We recall and apply the notions and notes which are given in [8].

Definition 1.1 Let $X$ be a linear space over $\mathbb{R}$ with $\operatorname{dim} X>1$, and let $\|\cdot, \cdot\|: X \times X \rightarrow \mathbb{R}$ be a function satisfying the following properties:

(1) $\|x, y\|=0$ if and only if $x, y$ are linearly dependent;

(2) $\|x, y\|=\|y, x\|$;

(3) $\|x, \alpha y\|=|\alpha|\|x, y\|$ for any $\alpha \in \mathbb{R}$;

(4) $\|x, y+z\| \leq\|x, y\|+\|x, z\|$;

for all $x, y, z \in X$ and $\alpha \in \mathbb{R}$. Then the function $\|\cdot, \cdot\|$ is called a 2-norm on $X$ and the pair $(X,\|\cdot, \cdot\|)$ is called a linear 2 -normed space.

Lemma 1.2 Let $(X,\|\cdot, \cdot\|)$ be a linear 2-normed space. If $x \in X$ and $\|x, y\|=0$ for all $y \in X$, then $x=0$.

Remark 1.3 Let $(X,\|\cdot, \cdot\|)$ be a linear 2-normed space. One can show that the conditions (2) and (4) in Definition 1.1 imply that

$$
|\|x, z\|-\|y, z\|| \leq\|x-y, z\|,
$$


for all $x, y, z \in X$. Hence, the functions $x \rightarrow\|x, y\|$ are continuous functions of $X$ into $\mathbb{R}$ for each fixed $y \in X$.

Definition 1.4 A sequence $\left\{x_{n}\right\}$ in a linear 2-normed space $X$ is called a Cauchy sequence if there are two linearly independent points $y, z \in X$ such that

$$
\lim _{m, n \rightarrow \infty}\left\|x_{n}-x_{m}, y\right\|=0 \quad \text { and } \quad \lim _{m, n \rightarrow \infty}\left\|x_{n}-x_{m}, z\right\|=0 .
$$

Definition 1.5 A sequence $\left\{x_{n}\right\}$ in a linear 2-normed space $X$ is called a convergent sequence if there is an $x \in X$ such that

$$
\lim _{n \rightarrow \infty}\left\|x_{n}-x, y\right\|=0
$$

for all $y \in X$. If $\left\{x_{n}\right\}$ converges to $\mathrm{x}$, write $x_{n} \rightarrow x$ as $n \rightarrow \infty$ and call $\mathrm{x}$ the limit of $\left\{x_{n}\right\}$. In this case, we also write $\lim _{n \rightarrow \infty} x_{n}=x$.

Lemma 1.6 For a convergent sequence $\left\{x_{n}\right\}$ in a linear 2-normed space $X$,

$$
\lim _{n \rightarrow \infty}\left\|x_{n}, y\right\|=\left\|\lim _{n \rightarrow \infty} x_{n}, y\right\|
$$

for all $y \in X$.

Definition 1.7 A linear 2-normed space in which every Cauchy sequence is a convergent sequence is called a 2-Banach space.

Hensel [9] has introduced a normed space which does not have the Archimedean property. During the last three decades, the theory of non-Archimedean spaces has gained the interest of physicists for their research in particular in problems coming from quantum physics, $p$-adic strings and superstrings [10]. Although many results in the classical normed space theory have a non-Archimedean counterpart, their proofs are different and require a rather new kind of intuition [11-14]. One may note that $|n| \leq 1$ in each valuation field, every triangle is isosceles and there may be no unit vector in a non-Archimedean normed space; $c f$. [12]. These facts show that the non-Archimedean framework is of special interest.

Definition 1.8 Let $\mathbb{K}$ be a field. A valuation mapping on $\mathbb{K}$ is a function $|\cdot|: \mathbb{K} \rightarrow \mathbb{R}$ such that for any $a, b \in \mathbb{K}$, we have

(i) $|a| \geq 0$ and the equality holds if and only if $a=0$,

(ii) $|a b|=|a||b|$,

(iii) $|a+b| \leq|a|+|b|$.

A field endowed with a valuation mapping will be called a valued field. If the condition (iii) in the definition of a valuation mapping is replaced with

$$
\text { (iii) }^{\prime} \quad|a+b| \leq \max \{|a|,|b|\},
$$

then the valuation $|\cdot|$ is said to be non-Archimedean. The condition (iii)' is called the strict triangle inequality. By (ii), we have $|1|=|-1|=1$. Thus, by induction, it follows 
from (iii)' that $|n| \leq 1$ for each integer $n$. We always assume in addition that $|\cdot|$ is nontrivial, i.e., there is an $a_{0} \in \mathbb{K}$ such that $\left|a_{0}\right| \notin\{0,1\}$. The most important examples of non-Archimedean spaces are $p$-adic numbers.

Example 1.9 Let $p$ be a prime number. For any non-zero rational number $a=p^{r} \frac{m}{n}$ such that $m$ and $n$ are coprime to the prime number $p$, define the $p$-adic absolute value $|a|_{p}=$ $p^{-r}$. Then $|\cdot|$ is a non-Archimedean norm on $\mathbb{Q}$. The completion of $\mathbb{Q}$ with respect to $|\cdot|$ is denoted by $\mathbb{Q}_{p}$ and is called the $p$-adic number field.

Definition 1.10 Let $X$ be a linear space over a scalar field $\mathbb{K}$ with a non-Archimedean non-trivial valuation $|\cdot|$. A function $\|\cdot\|: X \rightarrow \mathbb{R}$ is a non-Archimedean norm (valuation) if it satisfies the following conditions:

(NA1) $\|x\|=0$ if and only if $x=0$;

(NA2) $\|r x\|=|r|\|x\|$ for all $r \in \mathbb{K}$ and $x \in X$;

(NA3) the strong triangle inequality (ultrametric); namely, $\|x+y\| \leq \max \{\|x\|,\|y\|\}$

$$
(x, y \in X) \text {. }
$$

Then $(X,\|\cdot\|)$ is called a non-Archimedean normed space.

Now, we give the definition of a non-Archimedean 2-normed space which has been introduced in [15].

Definition 1.11 Let $X$ be a linear space with $\operatorname{dim} X>1$ over a scalar field $\mathbb{K}$ with a nonArchimedean non-trivial valuation $|\cdot|$. A function $\|\cdot, \cdot\|: X \rightarrow \mathbb{R}$ is a non-Archimedean 2-norm (valuation) if it satisfies the following conditions:

(NA1) $\|x, y\|=0$ if and only if $x, y$ are linearly dependent;

(NA2) $\|x, y\|=\|y, x\|$;

(NA3) $\|x, \alpha y\|=|\alpha|\|x, y\|$ for any $\alpha \in \mathbb{K}$;

(NA4) $\|x+y, z\| \leq \max \{\|x, z\|,\|y, z\|\}$;

for all $\alpha \in \mathbb{K}$ and $x, y, z \in X$. Then $(X,\|\cdot, \cdot\|)$ is called a non-Archimedean 2-normed space.

It follows from (NA4) that

$$
\left\|x_{m}-x_{l}, y\right\| \leq \max \left\{\left\|x_{J+1}-x_{J}, y\right\|: l \leq J \leq m-1\right\} \quad(m>l)
$$

and so a sequence $\left\{x_{m}\right\}$ is Cauchy in $X$ if and only if $\left\{x_{m+1}-x_{m}\right\}$ converges to zero in a non-Archimedean 2-normed space.

The stability problems concerning group homomorphisms were raised by Ulam [16] in 1940 and affirmatively answered for Banach spaces by Hyers [17] in the next year. The Hyers theorem was generalized by Rassias [18] for linear mappings by considering an unbounded Cauchy difference. In 1994, a generalization of the Rassias theorem was obtained by Gǎvruta [19] by replacing the unbounded Cauchy difference by a general control function.

In 2003, Radu [20] proposed a new method for obtaining the existence of exact solutions and error estimations, based on the fixed point alternative (see also [21, 22]).

Let $(X, d)$ be a generalized metric space. An operator $T: X \rightarrow X$ satisfies a Lipschitz condition with a Lipschitz constant $L$ if there exists a constant $L \geq 0$ such that $d(T x, T y) \leq$ $L d(x, y)$ for all $x, y \in X$. If the Lipschitz constant $L$ is less than one, then the operator $T$ 
is called a strictly contractive operator. Note that the distinction between the generalized metric and the usual metric is that the range of the former is permitted to include the infinity. We recall the following theorem by Margolis and Diaz.

Theorem 1.12 (cf. [20,23]) Suppose that we are given a complete generalized metric space $(\Omega, d)$ and a strictly contractive mapping $T: \Omega \rightarrow \Omega$ with the Lipschitz constant $L$. Then, for each given $x \in \Omega$, either

$$
d\left(T^{m} x, T^{m+1} x\right)=\infty \quad \text { for all } m \geq 0
$$

or there exists a natural number $m_{0}$ such that

- $d\left(T^{m} x, T^{m+1} x\right)<\infty$ for all $m \geq m_{0}$

- the sequence $\left\{T^{m} x\right\}$ is convergent to a fixed point $y^{*}$ of $T$;

- $y^{*}$ is the unique fixed point of $T$ in $\Lambda=\left\{y \in \Omega: d\left(T^{m_{0}} x, y\right)<\infty\right\}$;

- $d\left(y, y^{*}\right) \leq \frac{1}{1-L} d(y, T y)$ for all $y \in \Lambda$.

Khodaei and Rassias [24] investigated the solution and the Hyers-Ulam stability of an $n$-dimensional additive functional equation such that in the special case $n=2$,

$$
f(a x+b y)+f(a x-b y)=2 a f(x)
$$

for $a, b \in \mathbb{Z} \backslash\{0\}$ with $a \neq \pm 1, \pm b$. They proved that the Cauchy equation is equivalent to the above equation.

Jun and Kim [25] introduced the following cubic functional equation:

$$
f(2 x+y)+f(2 x-y)=2 f(x+y)+2 f(x-y)+12 f(x),
$$

and they established the general solution and the Hyers-Ulam stability for the functional equation (1.2). They proved that a mapping $f$ between two real vector spaces $X$ and $Y$ is a solution of (1.2) if and only if there exists a unique mapping $C: X \times X \times X \rightarrow Y$ such that $f(x)=C(x, x, x)$ for all $x \in X$. Moreover, $C$ is symmetric for each fixed one variable and is additive for fixed two variables. The mapping $C$ is given by $C(x, y, z)=\frac{1}{24}(f(x+y+z)+$ $f(x-y-z)-f(x+y-z)-f(x-y+z))$ for all $x, y, z \in X$. Obviously, the function $f(x)=c x^{3}$ satisfies the functional equation (1.2), which is called a cubic functional equation. Jun et al. [26] investigated the solution and the Hyers-Ulam stability for the cubic functional equation

$$
f(a x+b y)+f(a x-b y)=a b^{2}(f(x+y)+f(x-y))+2 a\left(a^{2}-b^{2}\right) f(x),
$$

where $a, b \in \mathbb{Z} \backslash\{0\}$ with $a \neq \pm 1, \pm b$.

Lee $e t$ al. [27] considered the following functional equation:

$$
f(2 x+y)+f(2 x-y)=4(f(x+y)+f(x-y))+24 f(x)-6 f(y) .
$$

In fact, they proved that a mapping $f$ between two real vector spaces $X$ and $Y$ is a solution of (1.4) if and only if there exists a unique symmetric bi-quadratic mapping $B_{2}: X \times X \rightarrow Y$ 
such that $f(x)=B_{2}(x, x)$ for all $x$. The bi-quadratic mapping $B_{2}$ is given by

$$
B_{2}(x, y)=\frac{1}{12}(f(x+y)+f(x-y)-2 f(x)-2 f(y)) .
$$

Obviously, the function $f(x)=a x^{4}$ satisfies the functional equation (1.4), which is called a quartic functional equation. Kang [28] investigated the Hyers-Ulam stability problem for the quartic functional equation

$$
\begin{aligned}
f(a x+b y)+f(a x-b y)= & a^{2} b^{2}(f(x+y)+f(x-y)) \\
& +2 a^{2}\left(a^{2}-b^{2}\right) f(x)-2 b^{2}\left(a^{2}-b^{2}\right) f(y),
\end{aligned}
$$

where $a, b \in \mathbb{Z} \backslash\{0\}$ with $a \neq \pm 1, \pm b$.

Recently, Ebadian, Najati and Eshaghi Gordji [29] considered the Hyers-Ulam stability of the systems of the additive-quartic functional equation

$$
\left\{\begin{array}{l}
f\left(x_{1}+x_{2}, y\right)=f\left(x_{1}, y\right)+f\left(x_{2}, y\right), \\
f\left(x, 2 y_{1}+y_{2}\right)+f\left(x, 2 y_{1}-y_{2}\right)=4 f\left(x, y_{1}+y_{2}\right)+4 f\left(x, y_{1}-y_{2}\right)+24 f\left(x, y_{1}\right)-6 f\left(x, y_{2}\right)
\end{array}\right.
$$

and the quadratic-cubic functional equation

$$
\left\{\begin{array}{l}
f\left(x, 2 y_{1}+y_{2}\right)+f\left(x, 2 y_{1}-y_{2}\right)=2 f\left(x, y_{1}+y_{2}\right)+2 f\left(x, y_{1}-y_{2}\right)+12 f\left(x, y_{1}\right), \\
f\left(x, y_{1}+y_{2}\right)+f\left(x, y_{1}-y_{2}\right)=2 f\left(x, y_{1}\right)+2 f\left(x, y_{2}\right) .
\end{array}\right.
$$

In this paper, we investigate the Hyers-Ulam stability for the system of the additivecubic-quartic functional equations

$$
\left\{\begin{array}{l}
f\left(a x_{1}+b x_{2}, y, z\right)+f\left(a x_{1}-b x_{2}, y, z\right)=2 a f\left(x_{1}, y, z\right), \\
f\left(x, a y_{1}+b y_{2}, z\right)+f\left(x, a y_{1}-b y_{2}, z\right) \\
=a b^{2}\left(f\left(x, y_{1}+y_{2}, z\right)+f\left(x, y_{1}-y_{2}, z\right)\right) \\
\quad+2 a\left(a^{2}-b^{2}\right) f\left(x, y_{1}, z\right), \\
f\left(x, y, a z_{1}+b z_{2}\right)+f\left(x, y, a z_{1}-b z_{2}\right) \\
=a^{2} b^{2}\left(f\left(x, y, z_{1}+z_{2}\right)+f\left(x, y, z_{1}-z_{2}\right)\right) \\
\quad+2 a^{2}\left(a^{2}-b^{2}\right) f\left(x, y, z_{1}\right)-2 b^{2}\left(a^{2}-b^{2}\right) f\left(x, y, z_{2}\right),
\end{array}\right.
$$

where $a, b \in \mathbb{Z} \backslash\{0\}$ with $a \neq \pm 1, \pm b$. The function $f: \mathbb{R} \times \mathbb{R} \times \mathbb{R} \rightarrow \mathbb{R}$ given by $f(x, y, z)=$ $c x y^{3} z^{4}$ is a solution of (1.6). In particular, letting $y=z=x$, we get an octic function $g: \mathbb{R} \rightarrow$ $\mathbb{R}$ in one variable given by $g(x):=f(x, x, x)=c x^{8}$. The proof of the following proposition is evident, and we omit the details.

Proposition 1.13 Let $X$ and $Y$ be real linear spaces. If a mapping $f: X^{3} \rightarrow Y$ satisfies (1.6), then $f(\lambda x, \mu y, \eta z)=\lambda \mu^{3} \eta^{4} f(x, y, z)$ for all $x, y, z \in X$ and all rational numbers $\lambda, \mu, \eta$.

We mention here the papers [30-35] concerning the Hyers-Ulam stability of the mixed type functional equations, the Hyers-Ulam stability in non-Archimedean Banach spaces and the Hyers-Ulam stability by fixed point methods. 
In the rest of this paper, unless otherwise explicitly stated, we will assume that $X$ is a non-Archimedean normed space and $Y$ is a non-Archimedean 2-Banach space.

\section{Approximation of octic mappings}

In this section, we investigate the Hyers-Ulam stability problem for the system (1.6) in non-Archimedean 2-Banach spaces.

Theorem 2.1 Let $s \in\{-1,1\}$ be fixed. Let $\phi_{1}, \phi_{2}, \phi_{3}: X^{4} \rightarrow[0, \infty)$ be functions such that

$$
\begin{aligned}
\Phi(x, y, z):= & \left|\frac{1}{2}\right| \max \left\{\left|a^{-4 s+3}\right| \phi_{1}\left(a^{\frac{s-1}{2}} x, 0, a^{\frac{s-1}{2}} y, a^{\frac{s-1}{2}} z\right)\left|a^{-4 s}\right| \phi_{2}\left(a^{\frac{s+1}{2}} x, a^{\frac{s-1}{2}} y, 0, a^{\frac{s-1}{2}} z\right),\right. \\
& \left.\left|a^{-4 s-4}\right| \phi_{3}\left(a^{\frac{s+1}{2}} x, a^{\frac{s+1}{2}} y, a^{\frac{s-1}{2}} z, 0\right)\right\}
\end{aligned}
$$

for all $x, y, z \in X$ and for some $0<L<1$,

$$
\Phi\left(a^{s} x, a^{s} y, a^{s} z\right) \leq L\left|a^{8 s}\right| \Phi(x, y, z)
$$

and

$$
\begin{aligned}
& \lim _{n \rightarrow \infty}\left|a^{-8 s n}\right| \phi_{1}\left(a^{s n} x_{1}, a^{s n} x_{2}, a^{s n} y, a^{s n} z\right)=0, \\
& \lim _{n \rightarrow \infty}\left|a^{-8 s n}\right| \phi_{2}\left(a^{s n} x, a^{s n} y_{1}, a^{s n} y_{2}, a^{s n} z\right)=0, \\
& \lim _{n \rightarrow \infty}\left|a^{-8 s n}\right| \phi_{3}\left(a^{s n} x, a^{s n} y, a^{s n} z_{1}, a^{s n} z_{2}\right)=0
\end{aligned}
$$

for all $x, y, z, x_{1}, x_{2}, y_{1}, y_{2}, z_{1}, z_{2} \in X$. Iff $: X^{3} \rightarrow Y$ is a mapping such that $f(x, y, 0)=0$ for all $x, y \in X$ and

$$
\begin{aligned}
& \left\|f\left(a x_{1}+b x_{2}, y, z\right)+f\left(a x_{1}-b x_{2}, y, z\right)-2 a f\left(x_{1}, y, z\right), u\right\| \leq \phi_{1}\left(x_{1}, x_{2}, y, z\right), \\
& \| f\left(x, a y_{1}+b y_{2}, z\right)+f\left(x, a y_{1}-b y_{2}, z\right)-a b^{2}\left(f\left(x, y_{1}+y_{2}, z\right)+f\left(x, y_{1}-y_{2}, z\right)\right) \\
& \quad-2 a\left(a^{2}-b^{2}\right) f\left(x, y_{1}, z\right), u \| \leq \phi_{2}\left(x, y_{1}, y_{2}, z\right), \\
& \| f\left(x, y, a z_{1}+b z_{2}\right)+f\left(x, y, a z_{1}-b z_{2}\right)-a^{2} b^{2}\left(f\left(x, y, z_{1}+z_{2}\right)+f\left(x, y, z_{1}-z_{2}\right)\right) \\
& \quad-2 a^{2}\left(a^{2}-b^{2}\right) f\left(x, y, z_{1}\right)+2 b^{2}\left(a^{2}-b^{2}\right) f\left(x, y, z_{2}\right), u \| \leq \phi_{3}\left(x, y, z_{1}, z_{2}\right),
\end{aligned}
$$

for all $u \in Y$ and $x, y, z, x_{1}, x_{2}, y_{1}, y_{2}, z_{1}, z_{2} \in X$, then there exists a unique octic mapping $T$ : $X \times X \times X \rightarrow Y$ satisfying (1.6) and

$$
\|f(x, y, z)-T(x, y, z), u\| \leq \frac{1}{1-L} \Phi(x, y, z)
$$

for all $u \in Y$ and $x, y, z \in X$.

Proof Putting $x_{1}=2 x$ and $x_{2}=0$ and replacing $y, z$ by $2 y, 2 z$ in (2.3), we get

$$
\|f(2 a x, 2 y, 2 z)-a f(2 x, 2 y, 2 z), u\| \leq\left|\frac{1}{2}\right| \phi_{1}(2 x, 0,2 y, 2 z)
$$


for all $u \in Y$ and $x, y, z \in X$. Putting $y_{1}=2 y$ and $y_{2}=0$ and replacing $x, z$ by $2 a x, 2 z$ in (2.4), we get

$$
\left\|f(2 a x, 2 a y, 2 z)-a^{3} f(2 a x, 2 y, 2 z), u\right\| \leq\left|\frac{1}{2}\right| \phi_{2}(2 a x, 2 y, 0,2 z)
$$

for all $u \in Y$ and $x, y, z \in X$. Putting $z_{1}=2 z$ and $z_{2}=0$ and replacing $x, y$ by $2 a x, 2 a y$ in (2.5), we get

$$
\left\|f(2 a x, 2 a y, 2 a z)-a^{4} f(2 a x, 2 a y, 2 z), u\right\| \leq\left|\frac{1}{2}\right| \phi_{3}(2 a x, 2 a y, 2 z, 0)
$$

for all $u \in Y$ and $x, y, z \in X$. Thus,

$$
\begin{aligned}
& \left\|f(2 a x, 2 a y, 2 a z)-a^{8} f(2 x, 2 y, 2 z), u\right\| \\
& \quad \leq\left|\frac{1}{2}\right| \max \left\{\left|a^{7}\right| \phi_{1}(2 x, 0,2 y, 2 z),\left|a^{4}\right| \phi_{2}(2 a x, 2 y, 0,2 z), \phi_{3}(2 a x, 2 a y, 2 z, 0)\right\}
\end{aligned}
$$

for all $u \in Y$ and $x, y, z \in X$. Replacing $x, y$ and $z$ by $\frac{x}{2}, \frac{y}{2}$ and $\frac{z}{2}$ in (2.10), we have

$$
\begin{aligned}
& \left\|f(a x, a y, a z)-a^{8} f(x, y, z), u\right\| \\
& \quad \leq\left|\frac{1}{2}\right| \max \left\{\left|a^{7}\right| \phi_{1}(x, 0, y, z),\left|a^{4}\right| \phi_{2}(a x, y, 0, z), \phi_{3}(a x, a y, z, 0)\right\}
\end{aligned}
$$

for all $u \in Y$ and $x, y, z \in X$. It follows from (2.11) that

$$
\begin{aligned}
& \left\|\frac{1}{a^{8}} f(a x, a y, a z)-f(x, y, z), u\right\| \\
& \quad \leq\left|\frac{1}{2}\right| \max \left\{\left|a^{-1}\right| \phi_{1}(x, 0, y, z),\left|a^{-4}\right| \phi_{2}(a x, y, 0, z),\left|a^{-8}\right| \phi_{3}(a x, a y, z, 0)\right\}
\end{aligned}
$$

and

$$
\begin{aligned}
& \left\|a^{8} f\left(\frac{x}{a}, \frac{y}{a}, \frac{z}{a}\right)-f(x, y, z), u\right\| \\
& \quad \leq\left|\frac{1}{2}\right| \max \left\{\left|a^{7}\right| \phi_{1}\left(\frac{x}{a}, 0, \frac{y}{a}, \frac{z}{a}\right),\left|a^{4}\right| \phi_{2}\left(x, \frac{y}{a}, 0, \frac{z}{a}\right), \phi_{3}\left(x, y, \frac{z}{a}, 0\right)\right\}
\end{aligned}
$$

for all $u \in Y$ and $x, y, z \in X$. From the inequalities (2.12) and (2.13), it follows that

$$
\left\|\frac{1}{a^{8 s}} f\left(a^{s} x, a^{s} y, a^{s} z\right)-f(x, y, z), u\right\| \leq \Phi(x, y, z)
$$

for all $u \in Y$ and $x, y, z \in X$.

Let $S$ be the set of all mappings $h: X \times X \times X \rightarrow Y$ with $h(x, y, 0)=0$ for all $x, y \in X$. And let us introduce a generalized metric on $S$ as follows:

$$
d(h, k)=\inf \left\{\alpha \in \mathbb{R}^{+}:\|h(x, y, z)-k(x, y, z), u\| \leq \alpha \Phi(x, y, z), \forall u \in Y \text { and } x, y, z \in X\right\},
$$


where, as usual, $\inf \emptyset=+\infty$. The proof of the fact that $(S, d)$ is a complete generalized metric space can be found in [21]. Now, we consider the mapping $J: S \rightarrow S$ defined by

$$
\operatorname{Jh}(x, y):=a^{-8 s} h\left(a^{s} x, a^{s} y, a^{s} z\right)
$$

for all $h \in S$ and $x, y, z \in X$. Let $f, g \in S$ such that $d(f, g)<\varepsilon$. Then

$$
\begin{aligned}
& \|J g(x, y, z)-J f(x, y, z), u\| \\
& \quad=\left\|a^{-8 s} g\left(a^{s} x, a^{s} y, a^{s} z\right)-a^{-8 s} f\left(a^{s} x, a^{s} y, a^{s} z\right), u\right\| \\
& \quad=\left|a^{-8 s}\right|\left\|g\left(a^{s} x, a^{s} y, a^{s} z\right)-f\left(a^{s} x, a^{s} y, a^{s} z\right), u\right\| \\
& \quad \leq\left|a^{-8 s}\right| \varepsilon \Phi\left(a^{s} x, a^{s} y, a^{s} z\right) \leq L \varepsilon \Phi(x, y, z),
\end{aligned}
$$

that is, if $d(f, g)<\varepsilon$, we have $d(J f, J g) \leq L \varepsilon$. This means that

$$
d(J f, J g) \leq L d(f, g)
$$

for all $f, g \in S$; that is, $J$ is a strictly contractive self-mapping on $S$ with the Lipschitz constant $L$. It follows from (2.14) that

$$
\|J f(x, y, z)-f(x, y, z), u\| \leq \Phi(x, y, z)
$$

for all $u \in Y$ and $x, y, z \in X$, which implies that $d(J f, f) \leq 1$. Due to Theorem 1.12, there exists a unique mapping $T: X \times X \times X \rightarrow Y$ such that $T$ is a fixed point of $J$, i.e., $T\left(a^{s} x, a^{s} y, a^{s} z\right)=a^{8 s} T(x, y, z)$ for all $x, y, z \in X$. Also, $d\left(J^{m} f, T\right) \rightarrow 0$ as $m \rightarrow \infty$, which implies the equality

$$
\lim _{m \rightarrow \infty} a^{-8 s m} f\left(a^{s m} x, a^{s m} y, a^{s m} z\right)=T(x, y, z)
$$

for all $x, y, z \in X$.

It follows from (2.1), (2.3), (2.4) and (2.5) that

$$
\begin{aligned}
\| & T\left(a x_{1}+b x_{2}, y, z\right)+T\left(a x_{1}-b x_{2}, y, z\right)-2 a T\left(x_{1}, y, z\right), u \| \\
= & \lim _{n \rightarrow \infty}\left|a^{-8 s n}\right| \| f\left(a^{s n} a x_{1}+a^{s n} b x_{2}, a^{s n} y, a^{s n} z\right)+f\left(a^{s n} a x_{1}-a^{s n} b x_{2}, a^{s n} y, a^{s n} z\right) \\
& -2 a f\left(a^{s n} x_{1}, a^{s n} y, a^{s n} z\right), u \| \\
\leq & \lim _{n \rightarrow \infty}\left|a^{-8 s n}\right| \phi_{1}\left(a^{s n} x_{1}, a^{s n} x_{2}, a^{s n} y, a^{s n} z\right)=0
\end{aligned}
$$

for all $u \in Y$ and $x_{1}, x_{2}, y, z \in X$,

$$
\begin{aligned}
\| T\left(x, a y_{1}+b y_{2}, z\right)+T\left(x, a y_{1}-b y_{2}, z\right)-a b^{2}\left(T\left(x, y_{1}+y_{2}, z\right)+T\left(x, y_{1}-y_{2}, z\right)\right) \\
\quad-2 a\left(a^{2}-b^{2}\right) T\left(x, y_{1}, z\right), u \| \\
=\lim _{n \rightarrow \infty} \frac{1}{a^{8 s n}} \| f\left(a^{s n} x, a^{s n} a y_{1}+a^{s n} b y_{2}, a^{s n} z\right)+f\left(a^{s n} x, a^{s n} a y_{1}-a^{s n} b y_{2}, a^{s n} z\right) \\
\quad-a b^{2}\left(f\left(a^{s n} x, a^{s n} y_{1}+a^{s n} y_{2}, a^{s n} z\right)+f\left(a^{s n} x, a^{s n} y_{1}-a^{s n} y_{2}, a^{s n} z\right)\right)
\end{aligned}
$$




$$
\begin{aligned}
& -2 a\left(a^{2}-b^{2}\right) f\left(a^{s n} x, a^{s n} y_{1}, a^{s n} z\right), u \| \\
\leq & \lim _{n \rightarrow \infty} \frac{1}{a^{8 s n}} \phi_{2}\left(a^{s n} x, a^{s n} y_{1}, a^{s n} y_{2}, a^{s n} z\right)=0
\end{aligned}
$$

for all $u \in Y$ and $x, y_{1}, y_{2}, z \in X$,

$$
\begin{aligned}
\| T\left(x, y, a z_{1}+b z_{2}\right)+T\left(x, y, a z_{1}-b z_{2}\right)-a^{2} b^{2}\left(T\left(x, y, z_{1}+z_{2}\right)+T\left(x, y, z_{1}-z_{2}\right)\right) \\
\quad-2 a^{2}\left(a^{2}-b^{2}\right) T\left(x, y, z_{1}\right)+2 b^{2}\left(a^{2}-b^{2}\right) T\left(x, y, z_{2}\right), u \| \\
=\lim _{n \rightarrow \infty}\left|a^{-8 s n}\right| \| f\left(a^{s n} x, a^{s n} y, a^{s n} a z_{1}+a^{s n} b z_{2}\right)+f\left(a^{s n} x, a^{s n} y, a^{s n} a z_{1}-a^{s n} b z_{2}\right) \\
\quad-a^{2} b^{2}\left(f\left(a^{s n} x, a^{s n} y, a^{s n} z_{1}+a^{s n} z_{2}\right)-f\left(a^{s n} x, a^{s n} y, a^{s n} z_{1}-a^{s n} z_{2}\right)\right) \\
\quad-2 a^{2}\left(a^{2}-b^{2}\right) f\left(a^{s n} x, a^{s n} y, a^{s n} z_{1}\right)+2 b^{2}\left(a^{2}-b^{2}\right) f\left(a^{s n} x, a^{s n} y, a^{s n} z_{2}\right), u \| \\
\leq \lim _{n \rightarrow \infty}\left|a^{-8 s n}\right| \phi_{3}\left(a^{s n} x, a^{s n} y, a^{s n} z_{1}, a^{s n} z_{2}\right)=0
\end{aligned}
$$

for all $u \in Y$ and $x, y, z_{1}, z_{2} \in X$. It follows from (2.15), (2.16) and (2.17) that $T$ satisfies (1.6), that is, $T$ is octic.

According to the fixed point alternative, since $T$ is the unique fixed point of $J$ in the set $\Omega=\{g \in S: d(f, g)<\infty\}, T$ is the unique mapping such that

$$
\|f(x, y, z)-T(x, y, z), u\| \leq \Phi(x, y, z)
$$

for all $u \in Y$ and $x, y, z \in X$. Using the fixed point alternative, we obtain that

$$
d(f, T) \leq \frac{1}{1-L} d(f, J f) \leq \frac{1}{1-L} \Phi(x, y, z)
$$

for all $x, y, z \in X$, which implies the inequality (2.6).

Remark 2.2 Let $X$ be a normed space and let $Y$ be a 2-Banach space in Theorem 2.1. Using the fixed point method, one can show that there exists a unique octic mapping $T: X^{3} \rightarrow Y$ satisfying (1.6) and

$$
\|f(x, y, z)-T(x, y, z), u\| \leq \frac{1}{1-L} \tilde{\Phi}(x, y, z)
$$

for all $u \in Y$ and $x, y, z \in X$, and

$$
\begin{aligned}
\tilde{\Phi}(x, y, z):= & \left|\frac{1}{2}\right|\left\{\left|a^{-4 s+3}\right| \phi_{1}\left(a^{\frac{s-1}{2}} x, 0, a^{\frac{s-1}{2}} y, a^{\frac{s-1}{2}} z\right)+\left|a^{-4 s}\right| \phi_{2}\left(a^{\frac{s+1}{2}} x, a^{\frac{s-1}{2}} y, 0, a^{\frac{s-1}{2}} z\right)\right. \\
& \left.+\left|a^{-4 s-4}\right| \phi_{3}\left(a^{\frac{s+1}{2}} x, a^{\frac{s+1}{2}} y, a^{\frac{s-1}{2}} z, 0\right)\right\}
\end{aligned}
$$

for all $x, y, z \in X$. It is easy to see that the approximation in non-Archimedean 2-normed spaces (inequality (2.6)) is better than the approximation in (Archimedean) 2-normed spaces (inequality (2.18)).

By the direct method, the following corollary is valid in the (Archimedean) Banach spaces. 
Corollary 2.3 Let $s \in\{-1,1\}$ be fixed and $\theta$, $p$ be nonnegative real numbers with $p s<8$, and let $X, Y$ be a normed space and a Banach space, respectively. Suppose that a mapping $f: X^{3} \rightarrow Y$ satisfying $f(x, y, 0)=0$ and

$$
\begin{aligned}
& \left\|f\left(a x_{1}+b x_{2}, y, z\right)+f\left(a x_{1}-b x_{2}, y, z\right)-2 a f\left(x_{1}, y, z\right)\right\| \leq \theta\left(\left\|x_{1}\right\|^{p}+\left\|x_{2}\right\|^{p}+\|y\|^{p}+\|z\|^{p}\right), \\
& \| f\left(x, a y_{1}+b y_{2}, z\right)+f\left(x, a y_{1}-b y_{2}, z\right)-a b^{2}\left(f\left(x, y_{1}+y_{2}, z\right)+f\left(x, y_{1}-y_{2}, z\right)\right) \\
& \quad-2 a\left(a^{2}-b^{2}\right) f\left(x, y_{1}, z\right) \| \leq \theta\left(\|x\|^{p}+\left\|y_{1}\right\|^{p}+\left\|y_{2}\right\|^{p}+\|z\|^{p}\right) \\
& \| f\left(x, y, a z_{1}+b z_{2}\right)+f\left(x, y, a z_{1}-b z_{2}\right)-a^{2} b^{2}\left(f\left(x, y, z_{1}+z_{2}\right)+f\left(x, y, z_{1}-z_{2}\right)\right) \\
& \quad-2 a^{2}\left(a^{2}-b^{2}\right) f\left(x, y, z_{1}\right)+2 b^{2}\left(a^{2}-b^{2}\right) f\left(x, y, z_{2}\right) \| \\
& \leq \theta\left(\|x\|^{p}+\|y\|^{p}+\left\|z_{1}\right\|^{p}+\left\|z_{2}\right\|^{p}\right)
\end{aligned}
$$

for all $x, y, z, x_{1}, x_{2}, y_{1}, y_{2}, z_{1}, z_{2} \in X$. Then there exists a unique octic mapping $T: X \times X \times$ $X \rightarrow Y$ satisfying

$$
\begin{aligned}
& \|f(x, y, z)-T(x, y, z)\| \\
& \quad \leq \frac{\theta a^{4(1-s)}}{2 s\left(a^{8}-|a|^{p}\right)}\left(\left(a^{7}+a^{4}|a|^{p}+|a|^{p}\right)\|x\|^{p}+\left(a^{7}+a^{4}+|a|^{p}\right)\|y\|^{p}+\left(a^{7}+a^{4}+1\right)\|z\|^{p}\right)
\end{aligned}
$$

for all $x, y, z \in X$.

The following example shows that the previous corollary is not valid in non-Archimedean Banach spaces.

Example 2.4 The most important examples of non-Archimedean spaces are $p$-adic numbers. A key property of $p$-adic numbers is that they do not satisfy the Archimedean axiom: for all $x$ and $y>0$, there exists an integer $n$ such that $x<n y$.

Let $p$ be a prime number. For any nonzero rational number $x$, there exists a unique integer $r \in \mathbb{Z}$ such that $x=\frac{a}{b} p^{r}$, where $a$ and $b$ are integers not divisible by $p$. Then $|x|_{p}:=p^{-r}$ defines a non-Archimedean norm on $\mathbb{Q}$. The completion of $\mathbb{Q}$ with respect to $|\cdot|$ is denoted by $\mathbb{Q}_{p}$ and is called the $p$-adic number field. Note that if $p \geq 3$, then $\left|2^{n}\right|_{p}=1$ for each integer $n$.

We consider the following special case of the system (1.6):

$$
\begin{aligned}
& \left\|f\left(2 x_{1}+x_{2}, y, z\right)+f\left(2 x_{1}-x_{2}, y, z\right)-4 f\left(x_{1}, y, z\right)\right\| \leq \theta\left(\left\|x_{1}\right\|^{r}+\left\|x_{2}\right\|^{r}+\|y\|^{r}+\|z\|^{r}\right), \\
& \left\|f\left(x, 2 y_{1}+y_{2}, z\right)+f\left(x, 2 y_{1}-y_{2}, z\right)-2\left(f\left(x, y_{1}+y_{2}, z\right)+f\left(x, y_{1}-y_{2}, z\right)\right)-12 f\left(x, y_{1}, z\right)\right\| \\
& \quad \leq \theta\left(\|x\|^{r}+\left\|y_{1}\right\|^{r}+\left\|y_{2}\right\|^{r}+\|z\|^{r}\right), \\
& \| f\left(x, y, 2 z_{1}+z_{2}\right)+f\left(x, y, 2 z_{1}-z_{2}\right)-4\left(f\left(x, y, z_{1}+z_{2}\right)+f\left(x, y, z_{1}-z_{2}\right)\right) \\
& \quad-24 f\left(x, y, z_{1}\right)+6 f\left(x, y, z_{2}\right) \| \leq \theta\left(\|x\|^{r}+\|y\|^{r}+\left\|z_{1}\right\|^{r}+\left\|z_{2}\right\|^{r}\right) .
\end{aligned}
$$

Let $X=Y=\mathbb{Q}_{p}$ for a prime number $p>5$ and define $f: X^{3} \rightarrow Y$ by $f\left(x_{1}, x_{2}, x_{3}\right)=x_{1} x_{2} x_{3}$. Then for $r=1, \theta=1$ and all $x_{1}, x_{2}, y_{1}, y_{2}, z_{1}, z_{2}, x, y, z \neq 0$ with $|x|_{p}<1,|y|_{p}<1,|z|_{p}<1$, we have

$$
\left|f\left(2 x_{1}+x_{2}, y, z\right)+f\left(2 x_{1}-x_{2}, y, z\right)-4 f\left(x_{1}, y, z\right)\right|_{p}=|0|_{p}=0 \leq\left|x_{1}\right|_{p}+\left|x_{2}\right|_{p}+|y|_{p}+|z|_{p},
$$


and

$$
\begin{aligned}
& \left|f\left(x, 2 y_{1}+y_{2}, z\right)+f\left(x, 2 y_{1}-y_{2}, z\right)-2\left(f\left(x, y_{1}+y_{2}, z\right)+f\left(x, y_{1}-y_{2}, z\right)\right)-12 f\left(x, y_{1}, z\right)\right|_{p} \\
& \quad=|x z|_{p} \cdot\left|-10 y_{1}\right|_{p} \leq\left|y_{1}\right|_{p} \leq|x|_{p}+\left|y_{1}\right|_{p}+\left|y_{2}\right|_{p}+|z|_{p},
\end{aligned}
$$

and

$$
\begin{aligned}
\mid f\left(x, y, 2 z_{1}+z_{2}\right)+f\left(x, y, 2 z_{1}-z_{2}\right)-4\left(f\left(x, y, z_{1}+z_{2}\right)+f\left(x, y, z_{1}-z_{2}\right)\right) \\
\quad-24 f\left(x, y, z_{1}\right)+\left.6 f\left(x, y, z_{2}\right)\right|_{p} \\
\quad=|x y|_{p} \cdot\left|-24 z_{1}+6 z_{2}\right|_{p} \leq \max \left\{\left|-24 z_{1}\right|_{p},\left|6 z_{2}\right|_{p}\right\} \\
\quad \leq \max \left\{\left|z_{1}\right|_{p},\left|z_{2}\right|_{p}\right\} \leq|x|_{p}+|y|_{p}+\left|z_{1}\right|_{p}+\left|z_{2}\right|_{p} .
\end{aligned}
$$

But for each natural number $n$, we have

$$
\begin{aligned}
& \left|2^{-8 s(n-1)} f\left(2^{s(n-1)} x_{1}, 2^{s(n-1)} x_{2}, 2^{s(n-1)} x_{3}\right)-2^{-8 s n} f\left(2^{s n} x_{1}, 2^{s n} x_{2}, 2^{s n} x_{3}\right)\right|_{p} \\
& \quad=\left|2^{-5 s(n-1)} x_{1} x_{2} x_{3}-2^{-5 s n} x_{1} x_{2} x_{3}\right|_{p} \\
& \quad \leq \max \left\{\left|2^{-5 s(n-1)} x_{1} x_{2} x_{3}\right|_{p},\left|-2^{-5 s n} x_{1} x_{2} x_{3}\right|_{p}\right\} \leq\left|x_{1} x_{2} x_{3}\right|_{p} .
\end{aligned}
$$

Hence, for each $x_{1}, x_{2}, x_{3} \neq 0,\left\{2^{-8 s n} f\left(2^{s n} x_{1}, 2^{s n} x_{2}, 2^{s n} x_{3}\right)\right\}$ is not convergent.

\section{Competing interests}

The authors declare that they have no competing interests.

\section{Authors' contributions}

All authors conceived of the study, participated in its design and coordination, drafted the manuscript, participated in the sequence alignment, and read and approved the final manuscript.

\section{Author details}

${ }^{1}$ Research Institute for Natural Sciences, Hanyang University, Seoul, 133-791, Korea. ${ }^{2}$ Department of Mathematics, Semnan University, P.O. Box 35195-363, Semnan, Iran. ${ }^{3}$ Department of Mathematics, Iran University of Science and Technology, Narmak, Tehran, Iran.

\section{Received: 29 January 2012 Accepted: 26 November 2012 Published: 10 December 2012}

\section{References}

1. Gähler, S: 2-metrische Räume und ihre topologische Struktur. Math. Nachr. 26, 115-148 (1963)

2. Gähler, S: Lineare 2-normierte Räumen. Math. Nachr. 28, 1-43 (1964)

3. Gähler, S: Über 2-Banach-Räume. Math. Nachr. 42, 335-347 (1969)

4. White, A: 2-Banach spaces. Doctorial Diss., St. Louis Univ. (1968)

5. White, A: 2-Banach spaces. Math. Nachr. 42, 43-60 (1969)

6. Lewandowska, Z: Linear operators on generalized 2-normed spaces. Bull. Math. Soc. Sci. Math. Roumanie (N.S.) 42, 353-368 (1999)

7. Lewandowska, Z: Bounded 2-linear operators on 2-normed sets. Glas. Mat. 39, 301-312 (2004)

8. Park, W: Approximate additive mappings in 2-Banach spaces and related topics. J. Math. Anal. Appl. 376, 193-202 (2011)

9. Hensel, K: Über eine neue Begründung der Theorie der algebraischen Zahlen. Jahresber. Dtsch. Math.-Ver. 6, 83-88 (1897)

10. Khrennikov, A: Non-Archimedean Analysis: Quantum Paradoxes, Dynamical Systems and Biological Models. Kluwer Academic, Dordrecht (1997)

11. Arriola, LM, Beyer, WA: Stability of the Cauchy functional equation over $p$-adic fields. Real Anal. Exch. 31, 125-132 (2005/2006)

12. Narici, L, Beckenstein, E: Strange terrain-non-Archimedean spaces. Am. Math. Mon. 88, 667-676 (1981)

13. Park, C, Boo, D, Rassias, TM: Approximately additive mappings over p-adic fields. J. Chungcheong Math. Soc. 21, 1-14 (2008) 
14. Vladimirov, VS, Volovich, IV, Zelenov, El: p-Adic Analysis and Mathematical Physics. World Scientific, River Edge (1994)

15. Freese, RW, Cho, Y: Geometry of Linear 2-Normed Spaces. Nova Science Publishers, Hauppauge (2001)

16. Ulam, SM: Problems in Modern Mathematics. Science edn. Wiley, New York (1964)

17. Hyers, DH: On the stability of the linear functional equation. Proc. Natl. Acad. Sci. USA 27, $222-224$ (1941)

18. Rassias, TM: On the stability of the linear mapping in Banach spaces. Proc. Am. Math. Soc. 72, 297-300 (1978)

19. Găvruta, P: A generalization of the Hyers-Ulam-Rassias stability of approximately additive mappings. J. Math. Anal. Appl. 184, 431-436 (1994)

20. Radu, V: The fixed point alternative and the stability of functional equations. Fixed Point Theory 4, 91-96 (2003)

21. Cădariu, L, Radu, V: On the stability of the Cauchy functional equation: a fixed point approach. Grazer Math. Ber. 346 43-52 (2004)

22. Cădariu, L, Radu, V: Fixed points and the stability of Jensen's functional equation. J. Inequal. Pure Appl. Math. 4, Article ID 4 (2003)

23. Margolis, B, Diaz, JB: A fixed point theorem of the alternative for contractions on the generalized complete metric space. Bull. Am. Math. Soc. 126, 305-309 (1968)

24. Khodaei, H, Rassias, TM: Approximately generalized additive functions in several variables. Int. J. Nonlinear Anal. Appl. $1,22-41(2010)$

25. Jun, K, Kim, H: The generalized Hyers-Ulam-Rassias stability of a cubic functional equation. J. Math. Anal. Appl. 274, 867-878 (2002)

26. Jun, K, Kim, H, Chang, I: On the Hyers-Ulam stability of an Euler-Lagrange type cubic functional equation. J. Comput. Anal. Appl. 7, 21-33 (2005)

27. Lee, S, Im, S, Hawng, I: Quartic functional equation. J. Math. Anal. Appl. 307, 387-394 (2005)

28. Kang, D: On the stability of generalized quartic mappings in quasi- $\beta$-normed spaces. J. Inequal. Appl. 2010, Article ID 198098 (2010)

29. Ebadian, A, Najati, A, Eshaghi Gordji, M: On approximate additive-quartic and quadratic-cubic functional equations in two variables on abelian groups. Results Math. 58, 39-53 (2010)

30. Eshaghi Gordji, M, Alizadeh, Z, Cho, Y, Khodaei, H: On approximate $C^{*}$-ternary m-homomorphisms: a fixed point approach. Fixed Point Theory Appl. 2011, Article ID 454093 (2011)

31. Eshaghi Gordji, M, Bavand Savadkouhi, M: On approximate cubic homomorphisms. Adv. Differ. Equ. 2009, Article ID 618463 (2009)

32. Eshaghi Gordji, M, Kaboli-Gharetapeh, S, Park, C, Zolfaghri, S: Stability of an additive-cubic-quartic functional equation. Adv. Differ. Equ. 2009, Article ID 395693 (2009)

33. Eshaghi Gordji, M, Karimi, T, Kaboli Gharetapeh, S: Approximately n-Jordan homomorphisms on Banach algebras. J. Inequal. Appl. 2009, Article ID 870843 (2009)

34. Eshaghi Gordji, M, Bavand Savadkouhi, M: Stability of a mixed type cubic and quartic functional equations in random normed spaces. J. Inequal. Appl. 2009, Article ID 527462 (2009)

35. Eshaghi Gordji, M, Bavand Savadkouhi, M, Park, C: Quadratic-quartic functional equations in RN-spaces. J. Inequal. Appl. 2009, Article ID 868423 (2009)

doi:10.1186/1029-242X-2012-289

Cite this article as: Park et al.: Fixed points and approximately octic mappings in non-Archimedean 2-normed spaces. Journal of Inequalities and Applications 2012 2012:289.

\section{Submit your manuscript to a SpringerOpen ${ }^{\circ}$ journal and benefit from:}

- Convenient online submission

Rigorous peer review

- Immediate publication on acceptance

- Open access: articles freely available online

- High visibility within the field

- Retaining the copyright to your article 